\title{
L'AZOTE AMMONIACAL, UN TOXIQUE POTENTIEL EN ÉLEVAGE DE POISSONS : LE CAS DU TURBOT.
}

\author{
J. PERSON-LE RUYET et G. BOEUF \\ IFREMER, Laboratoire de Physiologie des Poissons, Centre de Brest, \\ BP 70, 29280 Plouzane, France.
}

\section{RÉSUMÉ}

L'azote ammoniacal est un toxique potentiel en élevage de poissons ayant des effets plus ou moins sévères selon la concentration ambiante et la durée d'exposition. Dans une première partie, nous rappelons que l'AAT (somme de $\mathrm{NH}_{4}{ }^{+}$et $\mathrm{NH}_{3}$ ) est toxique essentiellement sous sa forme $\mathrm{NH}_{3}$. Dès qu'un poisson se trouve en présence de niveaux d'AAT anormalement élevés, il y a entrée d'AAT surtout sous forme de $\mathrm{NH}_{3}$ et diffusion dans les différents compartiments du poisson (à l'équilibre, le $\mathrm{NH}_{4}{ }^{+}$est dominant). Selon les conditions d'exposition, différents états d'équilibre se succèdent entre le poisson et le milieu dès lors que ses capacités d'excrétion sont affectées. A partir d'un certain niveau de concentration d'AAT dans le sang et les tissus, le poisson n'arrive plus à réguler ses fonctions vitales, le seuil de toxicité est atteint.

Nous faisons ensuite la synthèse de l'état des connaissances des effets de l'AAT sur la survie et la croissance du turbot (Psetta maxima) en le comparant à d'autres espèces de poissons élevées en mer ou en eau douce. A court terme, la sensibilité du turbot à l'AAT est du même ordre de grandeur que celle de la daurade et du bar, la 96-h CL50 (concentration létale pour $50 \%$ de la population) varie dans une fourchette de 1 à $3 \mathrm{mg} / / \mathrm{NH}_{3}$. Des valeurs aussi élevées ne peuvent être qu'accidentellement rencontrées, même dans les élevages à haute densité. Chez toutes les espèces marines éprouvées, le seuil de toxicité chronique est plus bas, 0,8-1 $\mathrm{mg} / \mathrm{l} \mathrm{NH}_{3}$ pour une durée d'exposition n'excédant pas un mois. Chez le turbot, la croissance est ralentie à $0,33 \mathrm{mg} / \mathrm{l} \mathrm{NH}$ en moyenne et sensiblement aux mêmes valeurs chez le bar et la daurade. Chez ces mêmes espèces selon la duré d'exposition, il y a risque de perturbation de la croissance au-dessus de $0,11 \mathrm{mg} / \mathrm{NH}_{3}$, ce qui correspond aux pics de concentrations dans les élevages à haute densité utilisant des circuits fermés. Ces seuils de sécurité en termes de croissance sont sensiblement plus bas chez les salmonidés $\left(0,02-0,15 \mathrm{mg} / \mathrm{NH}_{3}\right)$, surtout pendant la phase d'eau douce.

En définitive, dans des conditions de croissance par ailleurs optimales (facteurs du milieu et alimentation) le turbot et les poissons marins, pris au stade juvénile, sont relativement tolérants à l'AAT. Toutefois leur sensibilité est étroitement dépendante de facteurs environnementaux tels que le $\mathrm{pH}$ et la disponibilité en oxygène. Elle peut de même être modulée par les conditions d'exposition : pré-acclimatation, niveaux fluctuants d'AAT et durée d'exposition.

Mots-clés : azote ammoniacal, $\mathrm{NH}_{3}$, toxicité aiguë, toxicité chronique, poissons, turbot.

\section{AMMONIA, A POTENTIAL TOXIC TO FISH : APPLICATIONS TO TURBOT.}

\section{ABSTRACT}

Total ammonia nitrogen (TAN) is potentially toxic to fish, with moderate or severe effects on survival and growth depending on ambient levels and exposure durations. In a 
first point, the reasons why $\mathrm{NH}_{3}$ is the most toxic form of TAN (the sum of $\mathrm{NH}_{3}$ and $\mathrm{NH}_{4}{ }^{+}$) is developed. As soon as fish are exposed to abnormally high concentrations of exogenous TAN, $\mathrm{NH}_{3}$ immediately enters the fish and passively diffuses in all body compartments (at steady state, $\mathrm{NH}_{4}{ }^{+}$is the main form of TAN). Depending on time and ambient ammonia concentrations, various steady states are observed in relation with fish excretion capacity. Eventually, a threshold of blood plasma TAN and/or tissues contents is reached, above which all physiological functions are highly disturbed and death occurs (chronic lethal concentration).

In a second part, available data concerning acute and chronic toxicity of TAN to turbot (Psetta maxima) juveniles are summarized and compared with other fish species (marine or fresh water species). For short exposure, turbot tolerance to TAN is in the same range as in seabream and seabass, 96-h LC50s (lethal concentrations for $50 \%$ of the population) are 1-3 $\mathrm{mg} / / \mathrm{NH}_{3}$. Such high concentrations are unusual in fish farming even at high stocking densities. In comparison, chronic lethal concentrations are significantly lower, averaging 0.8-1 $\mathrm{mg} / \mathrm{l} \mathrm{NH}_{3}$ for one-month exposure duration. In all marine fish species, there is some risk of growth disturbances over $0.11 \mathrm{mg} / \mathrm{NH}_{3}$, usual concentrations in intensive fish farming using semi-closed systems. This safety level in terms of growth response is significantly lower in salmonids specially during fresh water rearing.

To conclude, under optimal rearing conditions (environmental factors and feeding conditions), turbot juveniles and other marine fish species tested are relatively tolerant to TAN. However, their sensitivity to TAN is highly dependent on environmental factors such as ambient $\mathrm{pH}$ and oxygen availability. Their tolerance may also be affected by exposure conditions : acclimation, fluctuating TAN levels and exposure duration.

Key-words : total ammonia nitrogen, $\mathrm{NH}_{3}$, acute toxicity, chronic toxicity, fish, turbot.

\section{INTRODUCTION}

Les contraintes économiques d'une aquaculture à la recherche d'une forte productivité imposent parfois l'utilisation de densités de poissons élevées et une réduction maximale des apports d'eau. Le corollaire est une diminution du potentiel de dilution des déchets produits par le catabolisme des poissons (composés azotés, $\mathrm{CO}_{2}$ ) et éventuellement par les excès d'aliments. Une diminution de la disponibilité en oxygène dissous est souvent associée à l'accumulation des composés azotés et à l'acidification du milieu, autant de freins potentiels à la réussite des élevages, d'où leur qualificatif de "facteurs limitants". C'est dans les élevages à terre utilisant des circuits fermés (apport d'eau neuve limité à 5-10\% par jour) que les risques de perturbations de la croissance sont les plus élevés, même si a priori des sécurités suffisantes ont été prises. Par ailleurs, nous ne pouvons exclure des difficultés inhérentes à la présence de niveaux élevés d'azote ammoniacal dans les élevages en cages de surface (faibles coefficients de marée et/ou absence de courant). L'optimisation et le dimensionnement des unités de recyclage reposent sur une meilleure connaissance des limites de tolérance des espèces élevées à ces facteurs limitants qui évoluent dans un système dynamique avec de fortes interactions entre eux. Une étape inévitable est l'étude des différents facteurs du milieu pris un à un (monofactoriel). Dans ce cadre, nous nous sommes intéressés à l'impact de l'azote ammoniacal sur la survie et la croissance des poissons marins.

Nous avons retenu comme principale espèce cible le turbot, Psetta maxima (Linné, 1758) ou Scophthalmus maximus (Rafinesque, 1810). C'est un poisson plat élevé en bassins dont la production aquacole vient d'émerger sur les marchés européens (3500 t en 1997 dont 1000 pour la France). Cette espèce benthique supporte l'entassement jusqu'à 4 couches successives, ce qui laisse envisager la possibilité d'utiliser des charges élevées 
pendant les deux années de grossissement, 75 à $110 \mathrm{~kg} / \mathrm{m}^{3}$ (JONES et al., 1981 ; PERSON-LE RUYET, 1993), et d'aménager des enceintes d'élevage spécifiques à l'espèce. Jusqu'à présent, la structure idéale pour le grossissement s'apparente à un bassin circulaire peu profond autonettoyant (CRIPPS et POXTON, 1992) et les charges pratiquées en phase finale dépassent rarement $50-60 \mathrm{~kg} / \mathrm{m}^{3}$ malgré une augmentation sensible ces dernières années. La tendance actuelle est à la recherche d'enceintes d'élevage profondes avec rayonnages, silos ou "raceways " profonds (LYGREN, 1994), ou au contraire extra-plates, " raceways " superposés (OIESTAD, 1995) permettant de réduire la surface d'implantation des fermes et de limiter les besoins en eau. Cette relative prudence dans l'intensification des élevages peut s'expliquer en partie par une méconnaissance des limites de tolérance de l'espèce aux facteurs limitants chez un poisson relativement réceptif aux agents pathogènes (TORANZO et al., 1994). II faut rappeler que jusqu'en 1996 (PERSON-LE RUYET et al., 1994 ; RASMUSSEN et KORSGAARD, 1996) les seules données sur les effets de l'azote ammoniacal et du $\mathrm{pH}$ chez le turbot provenaient d'un test préliminaire de 11 jours portant sur un effectif total de 33 poissons de $2 \mathrm{~g}$ (ALDERSON, 1979). A l'exception d'un " travail de pionnier " sur la sole, Solea solea, (ALDERSON, 1979), le seul rapprochement possible était avec les espèces d'eau douce, les salmonidés en particulier (HAYWOOD, 1983 ; MEADE, 1985 ; US EPA, 1989 ; RUSSO et THURSTON 1991 ; WOOD, 1993 ; TOMASSO, 1994).

Dans cette synthèse, nous rappelons pourquoi certaines formes d'azote ammoniacal sont toxiques et synthétiserons les résultats obtenus en toxicité aiguë et chronique chez le turbot juvénile en comparant sa sensibilité à celle d'autres poissons. Nous terminerons par une discussion sur les différents facteurs affectant la sensibilité des poissons à l'azote ammoniacal.

\section{GÉNÉRALITÉS SUR L'AZOTE AMMONIACAL}

\subsection{Différentes sources et formes de composés azotés}

Les principaux composés azotés présents dans le milieu d'élevage, azote ammoniacal et urée, sont essentiellement issus du catabolisme des protéines alimentaires. Chez les téléostéens, la proportion d'azote excrétée sous forme ammoniacale est prépondérante : selon les espèces et les conditions d'élevage, elle représente 40 à $80 \%$ de l'azote total excrété, la part de l'excrétion uréique étant de 5-25\% (RANDALL et WRIGHT, 1987 ; DOSDAT et al., 1996). A terme, l'azote ammoniacal est oxydé en nitrites, puis en nitrates par activité bactérienne et l'urée hydrolysée en azote ammoniacal et $\mathrm{CO}_{2}$. Des molécules plus complexes (acides aminés, créatinine, acide urique et oxyde de triméthylamine) sont aussi excrétées mais à des niveaux beaucoup plus faibles (RANDALL et WRIGHT, 1987). L'azote ammoniacal présent dans l'eau peut provenir aussi des excès d'aliments ou d'une contamination par des rejets industriels divers ou agricoles (fertilisants). De l'ensemble des déchets azotés, l'azote ammoniacal et les nitrites sont de loin les plus toxiques (revues de RUSSO et THURSTON, 1991 ; TOMASSO, 1994). Les risques de toxicité des nitrites sont réels lors de dysfonctionnement des systèmes de recyclage de l'eau; les seuils de toxicité aiguë (96-h CL50, se reporter au \$ 3.1) sont de 0,2-0,9 mg/l NO chez les poissons les plus sensibles, les salmonidés pendant la phase d'eau douce, et de $200 \mathrm{mg} / \mathrm{l}$ chez le bar européen à la salinité de $36 \mathrm{ppm}$. L'urée n'est pas toxique et les nitrates sont peu toxiques, les 96 -h CL50 sont de $1300 \mathrm{mg} / \mathrm{l} \mathrm{NO} \mathrm{N}_{3}$ chez les salmonidés.

Les niveaux d'azote ammoniacal rencontrés dans les élevages de poissons marins sont extrêmement variables selon les espèces et les conditions d'élevage. Ils se situent le plus souvent dans une fourchette de 0,007 à 1,8 mg/l (HANDY et POXTON, 1993) mais des valeurs de 4-5 mg/l d'AAT sont devenues courantes avec le développement d'élevages à haute densité. Des maximums de $8 \mathrm{mg} / \mathrm{l}$ sont ainsi observés chez le bar européen (Dicentrarchus labrax) élevé en circuit fermé à des densités d'élevage supérieures à $100 \mathrm{~kg} / \mathrm{m}^{3}$ (COVES et GASSET, 1996). A titre indicatif, les niveaux actuels autorisés pour 
les rejets en mer sont de $0,3 \mathrm{mg} / \mathrm{l}$ d'azote ammoniacal (HANDY et POXTON, 1993). La gestion des niveaux de composés azotés est relativement aisée dans les élevages à terre même si elle est plus délicate dans les systèmes fermés dont le bon fonctionnement repose sur l'équilibre de la flore bactérienne. L'amélioration de l'alimentation (rationnement), l'utilisation d'aliments à haute digestibilité et un prétraitement des matières en suspension avant la filtration biologique (filtration mécanique) contribuent efficacement à diminuer les risques de pollution azotée. Cette gestion des déchets azotés est plus difficile en cages et devient quasi impossible dans les systèmes d'élevage extensifs en l'absence de possibilité de régulation des débits d'eau. Toutefois, au vu des faibles densités pratiquées en élevage extensif, l'azote ammoniacal est rarement un facteur limitant.

\subsection{Propriétés physico-chimiques}

En solution aqueuse, l'azote ammoniacal se trouve sous deux formes: ammoniac libre ou moléculaire $\left(\mathrm{NH}_{3}\right)$ et ammoniac ionisé ou ion ammonium $\left(\mathrm{NH}_{4}{ }^{\circ}\right)$. L'azote ammoniacal (ammoniaque) est la somme de la forme ionisée et moléculaire, d'où l'utilisation du terme azote ammoniacal total (AAT). Pour des raisons de commodité de langage, dans cet article nous parlerons soit d'AAT, soit de $\mathrm{NH}_{3}$. Pour rappel, l'azote total est la somme de toutes les formes d'azote: $\mathrm{NH}_{3}, \mathrm{NH}_{4}, \mathrm{NO}_{2}, \mathrm{NO}_{3}$.

Le $\mathrm{NH}_{3}$ est très soluble dans l'eau et se comporte comme une base faible selon l'équation suivante dans sa forme étendue ou simplifiée :

$$
\begin{aligned}
\mathrm{NH}_{3}(\mathrm{~g})+\mathrm{nH}_{2} \mathrm{O}(\mathrm{l}) \Leftrightarrow & \mathrm{NH}_{3}-\mathrm{nH}_{2} \mathrm{O}(\mathrm{aq}) \Leftrightarrow \mathrm{NH}_{4}{ }^{+}+\mathrm{OH}+(\mathrm{n}-1) \mathrm{H}_{2} \mathrm{O}(\mathrm{l}) \\
& \mathrm{NH}_{3}+\mathrm{H}_{2} \mathrm{O} \Leftrightarrow \mathrm{NH}_{4}{ }^{+}+\mathrm{OH}^{+}
\end{aligned}
$$

La quantité relative des deux formes d'AAT est fortement influencée par des facteurs physiques qui sont par ordre d'importance : le $\mathrm{pH}$ du milieu, la température et la salinité. Des tables donnant les proportions de $\mathrm{NH}_{3} \mathrm{NH}_{4}{ }^{+}$ont été établies pour l'eau douce et l'eau de mer. Les mieux adaptées à l'eau de mer sont celles de JOHANSSON et WEDBORG (1980), AMINOT (1983). Le \% de $\mathrm{NH}_{3}$ par rapport à l'AAT, à partir des valeurs du $\mathrm{pH}$, de la température absolue $\left(\mathrm{T}(\mathrm{K})=\mathrm{t}\left({ }^{\circ} \mathrm{C}\right)+273,15\right)$ et de la salinité $\mathrm{S}$, se calcule à partir des équations suivantes :

$$
\begin{gathered}
\mathrm{NH}_{3}+\mathrm{H}^{+} \Leftrightarrow \mathrm{NH}_{4}^{+} \text {avec k1 }=\left[\mathrm{NH}_{4}^{+}\right] /\left[\mathrm{NH}_{3}\right]\left[\mathrm{H}^{+}\right] \\
\log \mathrm{k}_{1}=-0,467+0,00113 \times \mathrm{S}+2887,9 \times \mathrm{T}^{-1} . \\
\% \mathrm{NH}_{3}=\left[\mathrm{NH}_{3}\right] /\left[\mathrm{NH}_{4}{ }^{+}\right]+\left[\mathrm{NH}_{3}\right] \times 100 \\
\text { d'où \% } \mathrm{NH}_{3}=100 /\left[1+\exp _{10}\left(\text { logk }_{1}-\mathrm{pH}\right)\right] .
\end{gathered}
$$

Dans les élevages de poissons marins utilisant des $\mathrm{pH}(7,5-8,3)$ et des températures (16-22 ${ }^{\circ} \mathrm{C}$ ) élevés, les proportions de $\mathrm{NH}_{3}$ par rapport à l'AAT sont relativement fortes (Figure 1). A titre d'exemple, pour une température de $17^{\circ} \mathrm{C}$, une salinité de $35 \mathrm{ppm}$, le $\mathrm{NH}_{3}$ varie de 3,6 à 1,2\% de l'AAT lorsque le pH passe de 8,1 à 7,6. L'acidification du milieu liée à l'intensification des élevages ou à l'utilisation d'eau géothermale contribue ainsi à limiter les niveaux de $\mathrm{NH}_{3}$.

L'AAT est dosé par la méthode du bleu indophénol adaptée à la technique du flux continu segmenté pour Auto-Analyseur II Technicon. Dans sa version actuelle (DOSDAT et al., 1994), la précision est de $0,003 \mathrm{mg} / \mathrm{l}$ d'AAT dans une gamme de concentrations n'excédant pas $1,4 \mathrm{mg} / \mathrm{l}$ d'AAT.

\subsection{Propriétés biologiques de l'AAT (accumulation-élimination)}

Les échanges d'AAT entre le poisson et l'eau et vice versa se font essentiellement sous forme de $\mathrm{NH}_{3}$; c'est une molécule de petite taille, non chargée, qui diffuse aisément 


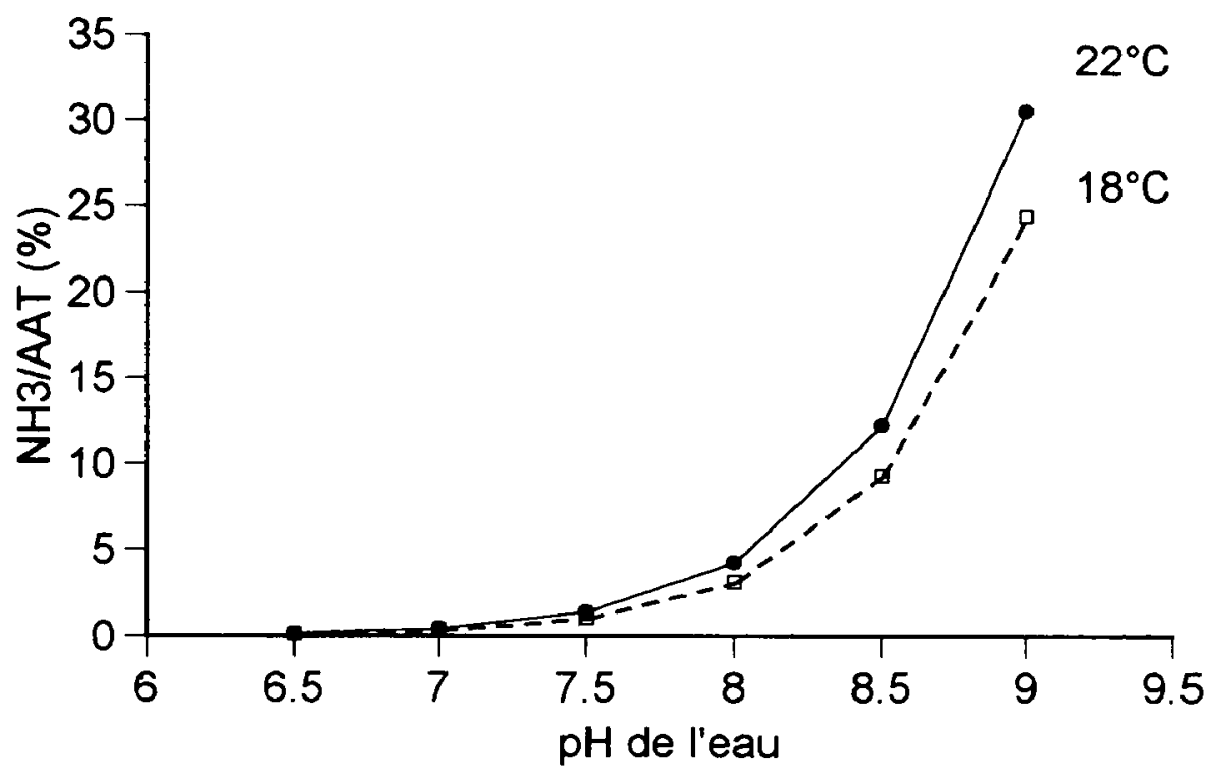

Figure 1

Proportion de $\mathrm{NH}_{3}(\%)$ dans l'azote ammoniacal total (AAT) en fonction du pH ambiant à 18 et $22^{\circ} \mathrm{C}$ pour une salinité de $35 \mathrm{ppm}$.

Figure 1

Percentage of $\mathrm{NH}_{3}$ in total ammonia nitrogen (TAN) related to ambient pH at 18 and $22{ }^{\circ} \mathrm{C}$ and 35 ppt salinity.

au travers des membranes riches en phospholipides. Le $\mathrm{NH}_{4}^{+}$ne peut pas pénétrer facilement les membranes lipidiques (par les canaux branchiaux) à cause de sa charge et de sa taille, même en eau de mer où la perméabilité branchiale au $\mathrm{NH}_{4}{ }^{+}$est plus élevée qu'en eau douce (WOOD, 1993). Ceci explique que le $\mathrm{NH}_{3}$ est considéré comme très toxique par rapport au $\mathrm{NH}_{4}{ }^{*}, 300$ à 400 fois plus, et que par convention la toxicité de l'AAT est exprimée en termes de $\mathrm{NH}_{3}$. La toxicité de l'AAT ne peut toutefois pas être uniquement attribuée à la forme $\mathrm{NH}_{3}$, le degré de toxicité des deux formes d'AAT variant selon le $\mathrm{pH}$ ambiant. Chez la crevette (Macrobrachium) et les salmonidés en eau douce, le $\mathrm{NH}_{3}$ serait surtout toxique aux $\mathrm{pH}$ élevés $\left(\mathrm{pH}>8\right.$ ) et le $\mathrm{NH}_{4}{ }^{+}$en milieu plus acide $(\mathrm{pH}<7)$ (ARMSTRONG et al., 1978 et THURSTON et al., 1981). A notre connaissance, aucune information n'est disponible en eau de mer.

Dès qu'un poisson est en présence d'une eau anormalement chargée en AAT, le $\mathrm{NH}_{3}$ diffuse immédiatement de l'eau ambiante vers le sang où il est protonisé en $\mathrm{NH}_{4}{ }^{+}$, ce qui maintient le gradient de pression partielle $(\mathrm{pNH} 3)$ entre l'eau et le sang. La diffusion dans les milieux extra- et intracellulaires du poisson est très rapide et se fait majoritairement sous forme de $\mathrm{NH}_{3}$, mais aussi sous forme de $\mathrm{NH}_{4}{ }^{+}$(WRIGHT et WOOD, 1985). Chez toutes les espèces étudiées, la cinétique d'entrée de l'AAT dans le poisson, obtenue par le dosage des teneurs plasmatiques, est relativement indépendante des niveaux d'AAT ambiant et, en dehors des concentrations létales, un premier état d'équilibre entre l'eau et le poisson (dépendant du niveau ambiant) est atteint en moins d'une heure, après la reprise de l'excrétion ammoniacale (CAMERON et HEISLER, 1983 ; WISE et al., 1989 ; PALEY et al., 1993 ; WILSON et al., 1994 ; PERSON-LE RUYET et al., 1998). Dès le retour à des concentrations en AAT faibles, l'élimination de l'AAT accumulé dans le poisson est immédiate et répond à la même cinétique que celle de l'entrée. La Figure 2 illustre les cinétiques d'entrée et d'évacuation d'AAT chez des turbots de $1 \mathrm{~kg}$ canulés à l'arc branchial. 
A

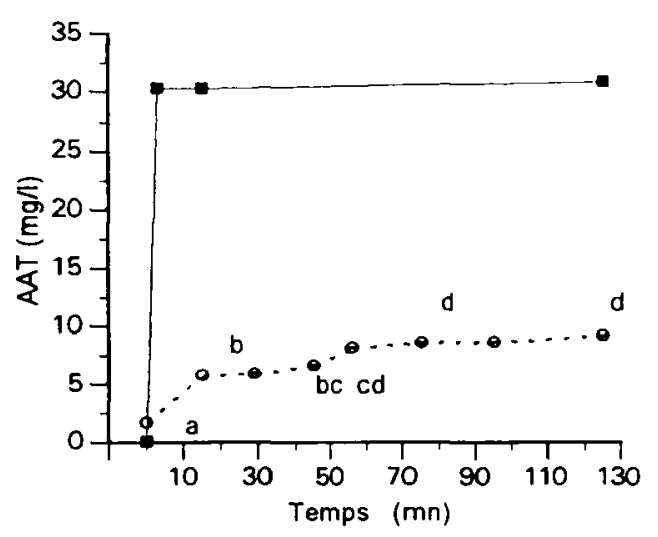

B

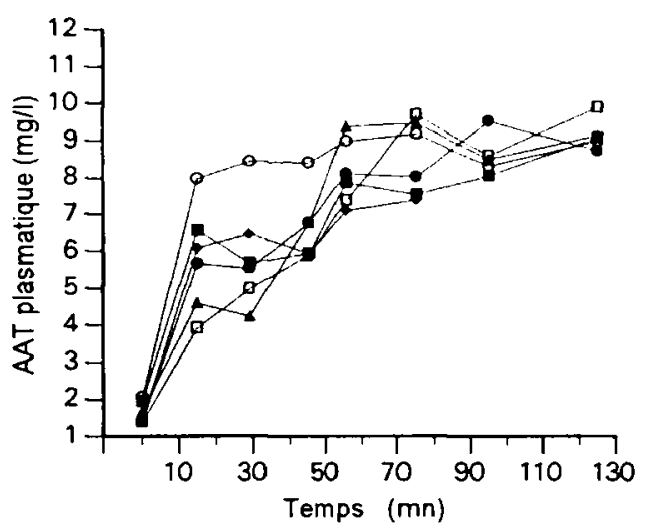

C

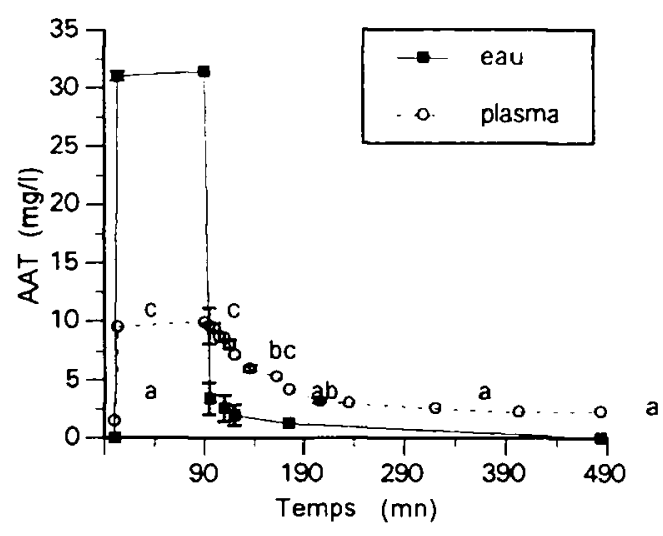

D

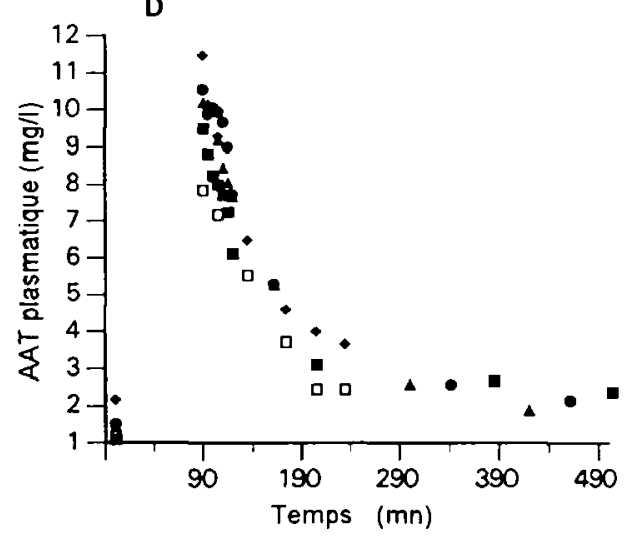

Figure 2

Cinétiques d'accumulation (2A) et d'élimination (2C) de I'AAT plasmatique en fonction des concentrations en AAT ambiant chez des turbots canulés et leur réponse individuelle pendant la phase d'entrée (2B) et d'évacuation (2D).

\section{Figure 2}

Kinetics of blood plasma TAN loading (2A) and unloading (2C) related to ambient TAN concentrations in cannulated turbot plus their individual responses in terms of plasma TAN contents during loading (2B) and unloading (2D).

Le niveau d'accumulation de l'AAT dans le plasma (l'ammoniémie) et les différents tissus est étroitement dépendant de la concentration ambiante (SCHENONE et al., 1982 ; SAHA et RATHA, 1990 ; PALEY et al., 1993 ; SAHA et al., 1994 ; BURY et al., 1995). Selon la concentration ambiante et la durée d'exposition, différents états d'équilibre entre le poisson et le milieu se succèdent au fur et à mesure que ses capacités d'excrétion s'amenuisent. Le gradient AAT ambiant/AAT sanguin diminue dans les stades précédant la mort, le poisson se laissant progressivement " envahir ". II y aurait une accumulation préférentielle dans certains tissus, qui évoluerait avec la durée d'exposition. Le cerveau semble être une des premières cibles, les concentrations en AAT pouvant y être 4 fois supérieures à celles du niveau précédant l'exposition (ARILLO et al., 1981). Fort heureusement, une protection relative du cerveau est mise en place rapidement (mécanismes de détoxification). Passée une période d'adaptation de quelques jours, si on en juge par des données obtenues chez un téléostéen d'eau douce, Heteropneustes fossilis, c'est dans le plasma et en particulier dans les érythrocytes que l'accumulation d'AAT est la plus forte et c'est dans le cerveau qu'elle est la plus faible (OGATA et MURAI, 
1988 ; SAHA et al., 1995). Le muscle, le foie et le rein sont aussi des sites de stockage important de l'AAT. Dans le plasma sanguin comme dans les autres tissus, I'AAT plasmatique est dosé par une méthode enzymatique basée sur la conversion du NADPH en NADP par le glutamate deshydrogénase à partir d'un “ kit Sigma UV ».

Avant de décrire la réponse des poissons à une ammoniémie élevée (en présence de concentrations ambiantes élevées) en termes d'excrétion ammoniacale, nous rappellerons comment elle se fait aux faibles concentrations en AAT ambiant. La voie préférentielle d'excrétion ammoniacale est la branchie, l'excrétion par la peau, les reins et les fèces étant secondaire et mal connue (RANDALL et WRIGHT, 1987). Les mécanismes d'excrétion sont assez controversés (EVANS et al., 1989 ; WOOD, 1993 ; WRIGHT, 1995) ; cependant, chez les poissons téléostéens, plusieurs voies sont possibles (Figure 3) :

SANG

BRANCHIE

EAU

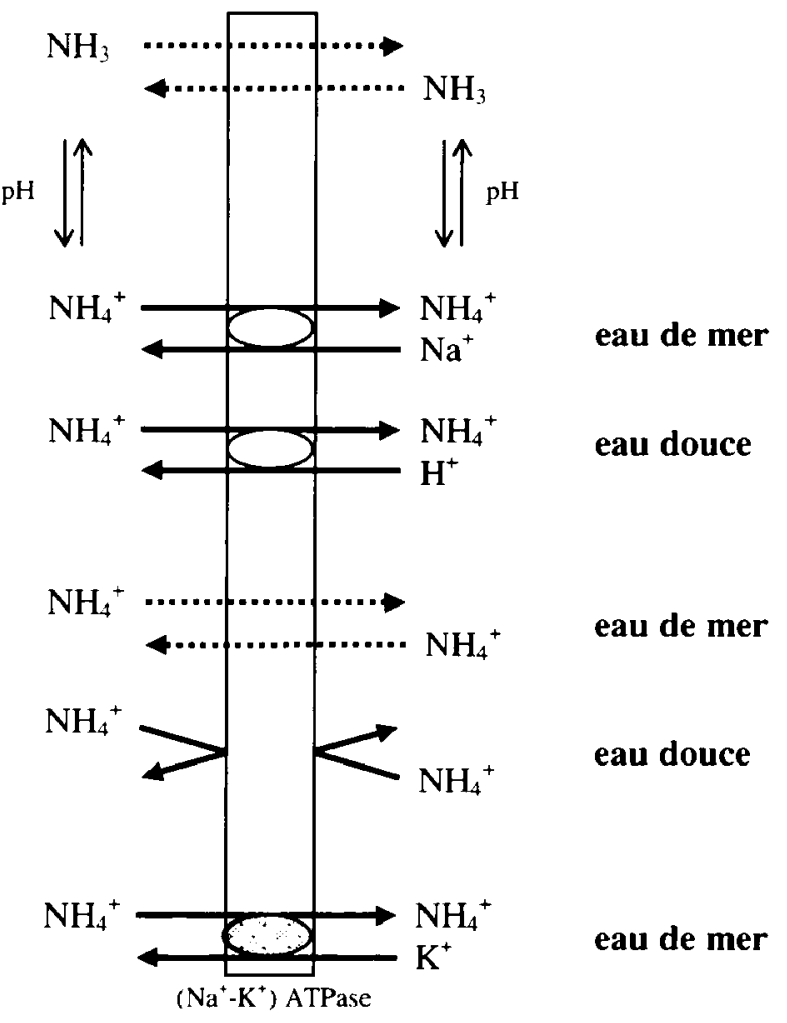

Figure 3

Mouvements possibles d'azote ammoniacal au travers de l'épithélium branchial : diffusion de $\mathrm{NH}_{3}$ suivant un gradient de pression partielle déterminée par les concentrations d'AAT et le pH de part et d'autre de la membrane branchiale ; excrétion active de $\mathrm{NH}_{4}^{+}$en échange de $\mathrm{Na}^{+}$et $\mathrm{K}^{+}$en eau de mer et de $\mathrm{H}^{+}$en eau douce ; diffusion limitée de $\mathrm{NH}_{4}{ }^{+}$en eau de mer et absence de diffusion de $\mathrm{NH}_{4}{ }^{+}$en eau douce.

\section{Figure 3}

Movements of ammonia across gill membrane : $\mathrm{NH}_{3}$ diffuses freely across the membrane in the direction favored by its pressure gradient which is determined by TAN concentrations and $\mathrm{pH}$ values on each side of the membrane. $\mathrm{NH}_{4}^{+}$can be transported outward by an active transport system in exchange for $\mathrm{Na}^{+}$and $\mathrm{K}^{+}$in sea water or $\mathrm{H}^{+}$in fresh water. Ammonium cannot diffuse accross the membrane in fresh water but it can partly diffuse in sea water. 
(1) une diffusion passive de $\mathrm{NH}_{3}$ au travers de l'épithélium branchial suivant un gradient de pression partielle du sang vers l'eau qui représenterait au minimum $70 \%$ de I'AAT excrété :

(2) une diffusion passive de $\mathrm{NH}_{4}$ selon un gradient électrochimique du sang vers l'eau qui serait relativement importante chez les poissons marins (jusqu'à $30 \%$ de l'AAT excrété) ;

(3) un échange électro-neutre $\mathrm{Na} / \mathrm{NH}_{;}$ou (4) $\mathrm{H}^{-} / \mathrm{NH}_{4}$ localisé sur les membranes apicales des cellules de l'épithélium branchial. II semblerait que ces deux mécanismes ne soient utilisés que lorsque les conditions d'excrétion deviennent difficiles (faible gradient $\left.\mathrm{pNH}_{3}\right)$;

(5) une substitution $\mathrm{K} / \mathrm{NH}_{4} \cdot$ faisant intervenir la $\left(\mathrm{Na}^{+}-\mathrm{K}^{+}\right)$-ATPase localisée au niveau de la membrane basolatérale serait de même possible (selon WOOD 1993) chez les poissons marins téléostéens.

L'exposition des poissons à des concentrations anormalement élevées d'AAT entraîne un blocage temporaire de l'excrétion ammoniacale suivi d'une reprise si les concentrations en AAT plasmatique n'atteignent pas des niveaux de toxicité aiguë. Chez les poissons exposés, la concentration en AAT plasmatique est toujours inférieure à celle de l'eau, ce qui laisse penser que l'excrétion se fait contre les gradients de pression partielle $\left(\mathrm{NH}_{3}\right.$ et $\left.\mathrm{NH}_{4}{ }^{\circ}\right)$. On a longtemps attribué la reprise de l'excrétion à un échange actif de $\mathrm{H}^{*}$ contre $\mathrm{NH}_{4}^{*}$ vers l'extérieur en eau douce et de $\mathrm{Na}^{+}$contre une excrétion de $\mathrm{NH}_{4}{ }^{-}$en eau de mer sans que ces deux hypothèses aient pu être validées expérimentalement (CAMERON et HEISLER, 1983 ; CAMERON, 1986 ; WILSON et TAYLOR, 1992). II est actuellement admis que l'excrétion se fait essentiellement par diffusion selon le gradient réel de $\mathrm{pNH}_{3}$ qui existe de part et d'autre de la branchie (EVANS et al., 1989 ; WRIGHT et al., 1989 ; WOOD, 1993 ; WILSON et al., 1994). En effet, le pH de l'eau à proximité immédiate de la branchie est légèrement plus bas (excrétion de $\mathrm{CO}_{2}$ ) que celui de l'ensemble de la masse d'eau, ce qui rend possible les échanges par diffusion même en présence de concentrations en AAT ambiant élevées.

\subsection{Mécanismes de toxicité et détoxification}

Les mécanismes de toxicité de l'AAT sont très mal connus chez les poissons. Par analogie avec les mammifères (pathologie humaine connue sous le nom d'encéphalopathie hépatique), l'AAT est considéré comme une neurotoxine du système nerveux central intervenant à la jonction du neurone synaptique et de la membrane lipoprotéique synaptique (COOPER et PLUM, 1987). C'est essentiellement la forme $\mathrm{NH}_{3}$ qui traverse la barrière sang-cerveau, avec quelques possibilités de passage de la forme $\mathrm{NH}_{4}{ }^{+}$qui pourrait alors entrer en compétition avec le $\mathrm{Na}^{+}$et le $\mathrm{K}^{+}$au niveau de la transmission de l'influx nerveux dans les axones.

Une exposition à de fortes concentrations en AAT ambiant se traduit par des perturbations métaboliques majeures au niveau cérébral. L'augmentation de l'ammoniémie provoque une augmentation des concentrations en glutamine (amide de l'acide glutamique transporteuse d'AAT), associée à une baisse des concentrations en glutamate (neuromédiateur excitateur de certains neurones du cortex cérébral) et en ATP (principale source d'énergie biologique). La glutamine est formée à partir du glutamate, la réaction étant catalysée par la glutamine synthétase (GS) en présence d'ATP (MOMMSEN et WALSH, 1992 ; TOMASSO, 1994). Le glutamate est issu de l'( $\iota$-cétoglutarate, la majorité des acides aminés servant de substrats dans cette réaction de transamination. II y a une augmentation très sensible de l'activité de la GS en réponse à une ammoniémie élevée. Elle n'est cependant pas suffisante pour éviter une accumulation d'AAT au niveau cérébral, il 
s'ensuit des réactions enzymatiques adaptatives en cascade coûteuses en énergie (répercussions importantes au niveau du cycle de Krebs). Les réactions initiales sont les suivantes :

$$
\begin{gathered}
\text { glutamate }+\mathrm{NH} 3 \Leftrightarrow \Leftrightarrow^{\text {gluamine synthetase (GS) }} \text { glutamine } \\
\alpha \text {-cétoglutarate } \Leftrightarrow \text { givarate deshydrogenase (GDH) } \\
\text { glutamate }
\end{gathered}
$$

Bien que réversibles, elles se déroulent préférentiellement dans le sens d'une diminution de l'ammoniémie. A ces effets métaboliques multiples de l'ammoniémie, il faut rajouter un déséquilibre, le plus souvent transitoire, de l'équilibre acide-base. L'alcalinisation cellulaire initiale $\left(\mathrm{NH}_{3}\right.$ est une base forte qui capte rapidement les $\left.\mathrm{H}^{+}\right)$ayant des répercussions sur l'activité de la plupart des enzymes. Des modifications structurales non-spécifiques de certains épithéliums (branchie, intestin, peau) contribuent de même à limiter les échanges poisson-milieu (LANG et al., 1987 ; GUILLEN et al., 1994 ; BANERJEE et BHATTACHARYA, 1995).

Aux concentrations létales, les manifestations secondaires de troubles neurologiques sévères sont une hyperexcitabilité, une hyperventilation, des mouvements désordonnés et violents avec perte d'équilibre et convulsions entraînant rapidement la mort. Pour des concentrations sublétales (n'entraînant pas de mortalité à court terme), ces troubles neurologiques ne sont pas perceptibles. Après une phase d'acclimatation de quelques jours à 2 semaines, aucune perturbation majeure des principales fonctions physiologiques (osmorégulation, respiration, nutrition) ne subsiste tant que les limites d'adaptation des poissons ne sont pas dépassées (KNOPH et THORUD, 1996 ; PERSON-LE RUYET et al., 1997a, 1998).

Les différences de tolérance à l'AAT entre espèces ou stades de développement seraient en rapport avec la capacité de détoxicification de l'AAT au niveau cérébral et plus précisément avec le taux d'activité spécifique de la GS du cerveau. C'est ce qui pourrait expliquer aussi les effets bénéfiques d'une pré-acclimatation. Parmi les nombreuses voies de détoxification de l'AAT, il faut citer aussi l'excrétion uréique. Chez de nombreuses espèces de poissons, la part de l'urée dans l'excrétion azotée est en effet augmentée lorsque l'excrétion ammoniacale devient extrêmement difficile, c'est le cas dans des eaux très alcalines ou très chargées en AAT (OLSON et FROMM, 1971 ; SAHA et RATHA, 1990 ; WRIGHT, 1993 ; SAHA et al., 1994 ; PERSON-LE RUYET et al., 1997a, b).

\section{LES SEUILS LÉTAUX CHEZ LES POISSONS (TOXICITÉ AIGUË)}

\subsection{Rappel du principe des tests CL50}

L'objectif des tests de toxicité aiguë est de déterminer, dans des conditions expérimentales standardisées (UNEP, 1989 ; ABEL et AXIAK, 1991), les limites de survie d'animaux calibrés en fonction de la concentration ambiante du produit testé. Les tests en flux continu, où le produit étudié est continuellement ajouté à l'arrivée d'eau, sont à préférer aux tests en statique (stabilité du $\mathrm{pH}$ et disponibilité en oxygène moins favorables). La durée de ces tests s'échelonne entre quelques heures et $96 \mathrm{~h}$ au maximum. A partir de l'évolution dans le temps de la mortalité observée pour chaque concentration du toxique (il faut au moins 5 concentrations donnant des mortalités significatives), on détermine la CL50, concentration ambiante se révélant létale pour $50 \%$ des individus exposés durant un temps d'exposition donné. Le TL50, temps nécessaire pour obtenir $50 \%$ de mortalité pour une concentration ambiante définie, est parfois utilisé en complément. Les procédures spécifiques de calculs sont détaillées dans plusieurs ouvrages dont ABEL et AXIAK (1991). Des logiciels spécifiques, le “ Toxicologist system " par exemple, sont disponibles. 
Le principal intérêt des CL50 est de comparer la sensibilité des différentes espèces ou stades de développement, de mesurer les effets de synergie et les antagonismes avec différents facteurs du milieu ou les effets d'un conditionnement préalable. Ils permettent aussi de quantifier les réponses physiologiques à court terme dans des conditions définies d'exposition et de déduire les DL50 (dose létale pour $50 \%$ de la population) de différents tissus pour certains indicateurs. Bien que la valeur prédictive des CL50 pour l'in situ soit discutable, elles sont très souvent à la base des premières extrapolations des seuils de sécurité en matière de protection de l'environnement ; ceux-ci sont estimés à 10 ou $5 \%$ de la 96-h CL50.

\subsection{CL50 chez le turbot}
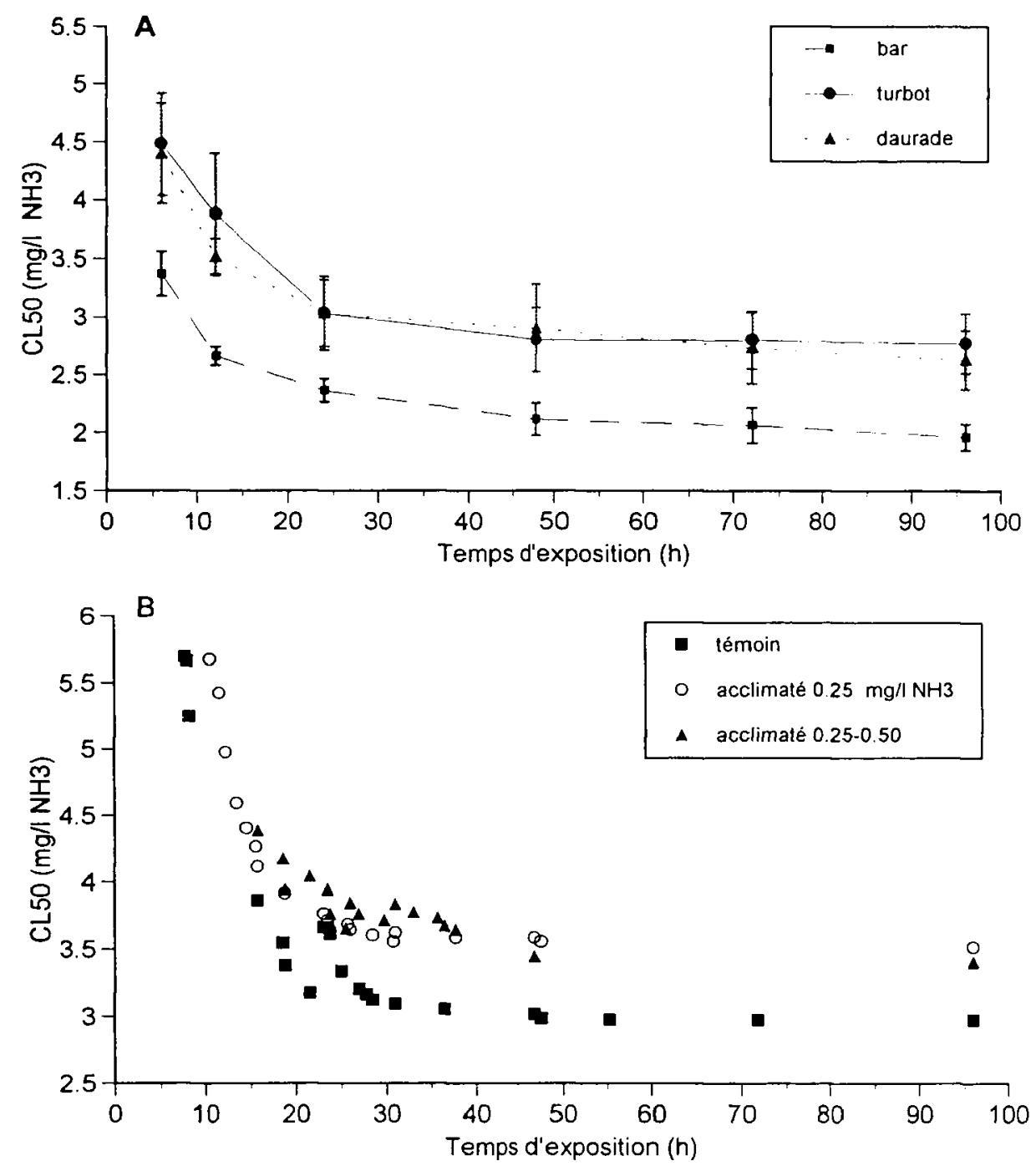

Figure 4

Evolution des CL50 (moyenne \pm es.) en fonction de la durée d'exposition (4A) chez des juvéniles de turbot $(n=5)$, bar $(n=6)$ et daurade $(n=3)$ et, $(4 B)$ chez des juvéniles de turbot pré-acclimatés ou non pendant un mois à 2 niveaux de $\mathrm{NH}_{3}$ ambiant $\left(17-18^{\circ} \mathrm{C} ; \mathrm{pH}, 8,1\right)$.

\section{Figure 4}

Changes in LC50s (mean \pm se) related to exposure duration $(4 A)$ in turbot $(n=5)$, seabass $(n=6)$ and seabream $(n=3)$ juveniles and, $(4 B)$ in turbot after or before an acclimation to low $\mathrm{NH}_{3}$ concentrations for one month $\left(17-18{ }^{\circ} \mathrm{C} ; \mathrm{pH}, 8.1\right)$. 
Les CL50 ont été déterminées en conditions standard $\left(17^{\circ} \mathrm{C}\right.$, oxygène $>80 \%$ de la saturation, $\mathrm{pH} 8,1$ ) avec des tests dynamiques chez 5 lots de turbot de 6 à $160 \mathrm{~g}$ en utilisant le $\mathrm{NH}_{4} \mathrm{Cl}$ comme source d'AAT (PERSON-LE RUYET et al., 1995). Elles diminuent lorsque la durée d'exposition augmente pour se stabiliser entre 48 et $96 \mathrm{~h}$ d'exposition autour de $2,5 \mathrm{mg} / \mathrm{l} \mathrm{NH}_{3}$ en moyenne, soit $56 \mathrm{mg} / \mathrm{l}$ d'AAT (Figure 4A). Aucun effet significatif de la taille n'a pu être mis en évidence malgré une tendance à une sensibilité accrue chez les plus gros poissons.

Lorsque des turbots de $25 \mathrm{~g}$ sont exposés à une concentration constante de $0,25 \mathrm{mg} / \mathrm{l}$ $\mathrm{NH}_{3}, 5$ jours par semaine pendant 4 semaines, leur aptitude à survivre à des chocs sévères de $\mathrm{NH}_{3}$ augmente sensiblement (Figure 4B). Par rapport à un lot témoin élevé à $0,002 \mathrm{mg} / \mathrm{l}$ $\mathrm{NH}_{3}$, la 96-h CL50 est majorée de $20 \%$ (3,6 au lieu de 3,0). Aucun gain n'est obtenu lorsque l'on y associe des pics journaliers de $0,50 \mathrm{mg} / \mathrm{l}$ de $\mathrm{NH}_{3}$ d'une durée de $7 \mathrm{~h}$.

\subsection{CL50 chez d'autres espèces}

Dans des conditions comparables de tests, $17-18^{\circ} \mathrm{C}$, la sensibilité à court terme du turbot est du même ordre que celle de la daurade dorée (Sparus aurata) et elle est plus faible que celle du bar européen (Tableau I). L'ensemble des résultats disponibles chez les juvéniles de poissons marins (WAJSBROT et al., 1991 ; WEIRICH et al., 1993 ; TOMASSO, 1993 ; PERSON-LE RUYET et al., 1995 ; BIANCHINI et al., 1996) montre que le turbot, la daurade et le bar sont plus résistants qu'un hybride de bar américain (Morone chrysops $\mathrm{X}$ $M$. saxatilis) et que le poisson plat Paralichthys orbignyanus, tous deux éprouvés à $25^{\circ} \mathrm{C}$. Pendant sa phase marine, le saumon atlantique (Salmo salar) a une 96-h CL50 de

\section{Tableau 1}

Comparaison des CL50 aiguës exprimées en $\mathrm{NH}_{3}$ et AAT (mg/l) chez des juvéniles de 5 espèces de poissons d'eau de mer et chez le saumon atlantique en eau douce et eau de mer.

Table 1

Comparative acute LC50 expressed as $\mathrm{NH}_{3}$ and TAN concentrations (mg/l) in juveniles of 5 marine fish species plus Atlantic salmon in fresh and sea water.

\begin{tabular}{|c|c|c|c|c|c|c|}
\hline \multirow{2}{*}{ Espèce } & \multirow{2}{*}{$\begin{array}{l}\text { Durée } \\
\text { (h) }\end{array}$} & \multirow{2}{*}{$\begin{array}{l}\text { Poids } \\
\text { (g) }\end{array}$} & \multicolumn{2}{|c|}{ CL50 (mg/l) } & \multirow{2}{*}{$\begin{array}{c}\text { Conditions } \\
\text { expérimentales }\end{array}$} & \multirow{2}{*}{ Références } \\
\hline & & & $\mathbf{N H}_{3}$ & AAT & & \\
\hline $\begin{array}{c}\text { Turbot } \\
\text { (Psetta maxima) }\end{array}$ & 96 & 6.163 & $\begin{array}{c}1,5-3,2 \\
(2,5)\end{array}$ & $35 \cdot 74$ & $\begin{array}{c}18^{\circ} \mathrm{C}, \mathrm{pH} 8,15, \mathrm{~S}: 35 \\
\text { flux continu }\end{array}$ & PERSON-LE RUYET et al., 1995 \\
\hline \multirow{2}{*}{$\begin{array}{l}\text { Daurade dorée } \\
\text { (Sparus aurata) }\end{array}$} & 96 & $0.4-3$ & 1,3 & 23.7 & $\begin{array}{c}26^{\circ} \mathrm{C}, \mathrm{pH} 8,01, \mathrm{~S}: 40 \\
\text { stagnant }\end{array}$ & WAJSBROT et al., 1991 \\
\hline & 96 & $6 \cdot 136$ & $\begin{array}{c}2,2-2,9 \\
(2,6)\end{array}$ & $51-67$ & $\begin{array}{c}18^{\circ} \mathrm{C}, \mathrm{pH} 8,15, \mathrm{~s}: 34 \\
\text { stagnant }\end{array}$ & PERSON-LE RUYET et al., 1995 \\
\hline $\begin{array}{c}\text { Bar européen } \\
\text { (Oicentrarchus } \\
\text { labrax) }\end{array}$ & 96 & $6-93$ & $\begin{array}{l}1 \cdot 2,3 \\
(1,7)\end{array}$ & $23-51$ & $\begin{array}{c}18 \mathrm{C}, \mathrm{pH} 8,15, \mathrm{~S}: 34 \\
\text { flux continu }\end{array}$ & PERSON-LE RUYET et al. 1995 \\
\hline $\begin{array}{c}\text { Bar américain } \\
\text { hybride } \\
\text { (Morone sp) }\end{array}$ & 96 & 43 & 0.7 & - & $\begin{array}{c}25^{\circ} \mathrm{C}, \mathrm{pH} 7,3, \mathrm{~S}: 35 \\
\text { stagnant }\end{array}$ & WEIRICH and TOMASSO, 1993 \\
\hline \multirow[t]{2}{*}{$\begin{array}{c}\text { Cardeau } \\
\text { (Paralichthys } \\
\text { orbignyanus) }\end{array}$} & 96 & 120 & 0.7 & 49.6 & $\begin{array}{l}25^{\circ} \mathrm{C},-, \mathrm{S}: 30 \\
\text { stagnant }\end{array}$ & BIANCHINI et al. 1996 \\
\hline & 48 & 430 & $0.34-0.24$ & 59 & $\begin{array}{c}6^{\circ} \mathrm{C}, \mathrm{pH} 7,55, \mathrm{~S}: 34 \\
\text { flux continu }\end{array}$ & KNOPH et THORUD, 1996 \\
\hline \multirow[t]{2}{*}{$\begin{array}{l}\text { Saumon attantique } \\
\text { (Salmo salar) }\end{array}$} & 24 & $50-100$ & 0.18 & - & $\begin{array}{c}10^{\circ} \mathrm{C}, \mathrm{pH} 8,1, \mathrm{~S}: 0 \\
\text { stagnant }\end{array}$ & ALABASTER ot al. 1983 \\
\hline & 96 & $10-15$ & $0,03-0,15$ & $319-239$ & $\begin{array}{c}2-12{ }^{\circ} \mathrm{C}, \mathrm{pH} 6,2, \mathrm{~S}: 0 \\
\text { stagnant }\end{array}$ & KNOPH, 1992 \\
\hline
\end{tabular}


0,24-0,34 mg/l NH3; en eau douce, la sensibilité des jeunes stades est plus élevée (ALABASTER et al., 1983 ; KNOPH, 1992 ; KNOPH et THORUD, 1996).

L'ensemble des données, existant sur la toxicité aiguë du $\mathrm{NH}_{3}$ chez les poissons d'intérêt commercial, montre que sur une échelle de sensibilité on a aux deux extrémités: les poissons-chats et les salmonidés (RUFFIER et al., 1981 ; US EPA, 1989 ; HAYWOOD, 1983 ; RUSSO et THURSTON, 1991). Les salmonidés sont les plus sensibles, les 96-h CL50 se situent dans une fourchette $0,07-0,90 \mathrm{mg} / / \mathrm{NH}_{3}$. Les poissons marins tempérés éprouvés (Tableau I) ainsi que l'esturgeon sibérien, Acipenser baeri, (SALIN et WILLIOT, 1991) seraient du type poisson-chat, relativement tolérants au $\mathrm{NH}_{3}$ pour de courtes périodes. Les CL50 correspondent à des valeurs excessivement élevées, 10-20 fois les $\mathrm{NH}_{3}$ maximums rencontrés en pisciculture marine ; sauf accident majeur, il y a donc peu de risques de toxicité aiguë de l'AAT. A titre comparatif, les 96-h CL50 sont de l'ordre de 0,4-2,3 mg/l $\mathrm{NH}_{3}$ pour les crustacés et 3,3-6,0 pour les mollusques (RUFFIER et al., 1981).

\section{LES SEUILS DE TOXICITÉ CHRONIQUE CHEZ LES POISSONS}

\subsection{Tests de croissance}

Par rapport à la toxicité aiguë, la toxicité chronique traite des effets à plus long terme de concentrations " sublétales ", c'est-à-dire n'engendrant pas de mortalité immédiate au niveau d'une population. Les tests biologiques globaux sont les plus utilisés (ABEL et AXIAK, 1991). Ils sont impérativement réalisés en milieu renouvelé, la principale difficulté étant de maintenir les conditions expérimentales constantes sur de longues périodes, un à plusieurs mois. Ils sont aussi basés sur la bonne interprétation des réponses des animaux au produit testé, ce qui impose de les placer dans un environnement contrôlé où la croissance est optimale en l'absence de polluant. Les tests in vitro, d'interprétation plus délicate, ne sont pas, à notre connaissance, actuellement utilisés pour l'AAT.

Les tests biologiques évaluent les diverses modifications (perturbations du comportement, de la croissance, de la reproduction, du développement larvaire, ou celles d'une fonction physiologique définie ou d'une activité enzymatique donnée) provoquées par l'exposition au produit étudié afin d'estimer les risques encourus dans des conditions de concentrations et de durée d'exposition précises. Les CL50 chroniques sont déterminées ainsi que les " seuils de risque " (LOEC : concentration la plus faible ayant un effet), et les seuils de "sécurité " (NOEC : concentration la plus forte n'ayant pas d'effet). Ces différents seuils varient selon le critère retenu (croissance, appétit, efficacité alimentaire) et n'ont qu'une valeur indicative, même lorsque la durée minimale recommandée, un mois, est respectée (KOOIJMAN et BEDAUX, 1996). Des tests plus courts, 2 semaines, recouvrent la période d'adaptation des poissons au polluant et conduisent à une surestimation de leur sensibilité. II faudrait disposer d'indicateurs plus sensibles (indicateurs prédictifs de croissance) sur la base d'une réponse de très faible amplitude indépendamment de sa significativité sur un plan statistique (HOEKSTRA et VAN EWIJK, 1993).

\subsection{Seuils de survie et de croissance chez le turbot}

Les effets d'une concentration constante de $\mathrm{NH}_{3}$ ont été testés à 3 périodes de l'année chez 5 lots de turbot d'origine différente (poids moyen, 23-104 g). Les résultats de croissance et de survie, obtenus pour une durée d'exposition de 28 à 84 jours, sont récapitulés dans le Tableau II (PERSON-LE RUYET et al., 1997a, b). Aucune mortalité n'a été observée jusqu'à $0,35 \mathrm{mg} / \mathrm{l} \mathrm{NH}_{3}$. Des taux de mortalité de $50 \%$ sont enregistrés en 28 jours à $1 \mathrm{mg} / \mathrm{l}$ en moyenne (extrêmes, $0,8-1,2$ ) contre 52 ou 72 jours à $0,70 \mathrm{mg} / \mathrm{l} \mathrm{NH} \mathrm{H}_{3}$. Le 
seuil de toxicité chronique (limite de survie pour $50 \%$ de la population) est étroitement dépendant de la durée d'exposition, il correspond en moyenne à $35 \%$ de la 96 -h CL50 pour une durée d'exposition de 28 jours.

Chez le turbot, la croissance est inhibée à partir de $0,82 \mathrm{mg} / \mathrm{l} \mathrm{NH}$ et elle est réduite de moitié dans une fourchette de 0,5 à 0,70 , l'impact de la durée d'exposition étant peu marqué à ces fortes concentrations. II n'en est pas de même aux plus faibles concentrations. Ainsi pour une durée d'exposition de 28 jours, la croissance est significativement ralentie à $0,33 \mathrm{mg} / / \mathrm{NH}_{3}$ en moyenne, soit 0,10 pour les lots les plus sensibles et 0,70 pour les plus tolérants. Pour des expositions prolongées, le seuil de perturbation de la croissance est abaissé : lors d'une expérience de 3 mois, et dans les conditions de concentrations (progression géométrique d'ordre 2) utilisées, il passe ainsi de $0,73-0,34 \mathrm{mg} / \mathrm{NH}_{3}$ à un mois à $0,33-0,17$ à 3 mois. La gamme de concentrations utilisée n'a permis de déterminer les seuils de sécurité en termes de croissance que pour les 2 lots de turbot les moins sensibles. Ainsi, en aucun cas, la sécurité des élevages n'est garantie au-dessus de 0,14-0,18 mg/l $\mathrm{NH}_{3}$ (en moyenne, 5-5,5 mg/l AAT). Cet exemple illustre les difficultés inhérentes à l'établissement de seuils de risque avec un maximum de sécurité même dans des conditions expérimentales favorables. Ce qui fait dire à KOOIJMAN et BEDAUX (1996) que " moins on en sait sur une espèce, plus les seuils de perturbation sont élevés ". Il est certain que passée une période d'adaptation de 2 semaines environ, plus la durée d'exposition est courte plus ces seuils sont élevés.

Chez des poissons nourris à la demande, la baisse des performances de croissance liée à la présence de niveaux de $\mathrm{NH}_{3}$ ambiant anormalement élevés est toujours associée à une réduction du taux d'alimentation. L'ampleur de la perte d'appétit et la vitesse de récupération d'un appétit normal, ou relatif, sont les meilleurs indicateurs de l'intensité du stress lié à une augmentation brutale de la concentration en AAT. Une baisse du taux d'alimentation se traduit à terme de 3 mois par une dégradation des capacités du poisson à utiliser l'aliment consommé : le taux de conversion alimentaire augmente et le pourcentage de protéines fixées diminue. La composition biochimique des poissons n'est modifiée (augmentation de la teneur en eau) que lors de perturbations majeures de la prise de nourriture.

\subsection{AAT plasmatique, un indicateur de toxicité de l'AAT chez le turbot}

La présence d'AAT dans le milieu d'élevage entraîne, selon la concentration et la durée d'exposition, des perturbations modérées ou extrêmement sévères, transitoires ou au contraire durables, de l'ensemble des fonctions physiologiques (respiration, osmorégulation, excrétion, croissance, reproduction, réaction immunitaire, etc.) ainsi que des altérations de nombreux tissus (branchie, intestin, peau, foie, etc.). Parmi les différents descripteurs de ces perturbations que nous avons testés chez le turbot à l'échelle de l'individu comme de la population, la teneur en AAT plasmatique est l'un des meilleurs (PERSON-LE RUYET et al., 1997a, b, 1998).

La concentration en AAT plasmatique est positivement corrélée à la concentration ambiante. Le turbot ne peut survivre à des doses de $40 \mathrm{mg} / \mathrm{l}$ pendant plus de 4 jours (seuils de toxicité aiguë), ces niveaux représentent plus de 20 fois le niveau de base de poissons non exposés à jeun depuis 6 jours. Des niveaux d'AAT plasmatique plus bas peuvent être tolérés pendant de plus longues périodes : $30-35 \mathrm{mg} / \mathrm{l}$ pendant $14-28$ jours et $20 \mathrm{mg} / \mathrm{l}$ pendant 3 mois (turbot à jeun depuis $24 \mathrm{~h}$ ). Dans nos conditions expérimentales, aucune mortalité n'a été observée jusqu'à $15 \mathrm{mg} / \mathrm{l}$, soit 5 fois plus que le niveau de base. Le niveau de perturbation de la croissance pour des durées d'exposition de 28 jours est d'environ $10 \mathrm{mg} / \mathrm{l}$ d'AAT. En dessous, les niveaux d'AAT plasmatique sont supérieurs aux concentrations ambiantes (le gradient poisson-eau est inversé) et l'excrétion de $\mathrm{NH}_{3}$ du poisson vers l'extérieur ne présente pas de difficulté particulière. Sous réserve d'une meilleure 
connaissance des contraintes et des limites d'utilisation de cet indicateur, il semble qu'il puisse avoir une valeur prédictive des seuils de perturbation de la croissance et de la survie chez d'autres espèces que le turbot (LUMSDEN et al., 1993 ; CHO et al., 1994 ; KNOPH et THORUD, 1996).

\subsection{Seuils de survie et de croissance chez les autres poissons}

La synthèse de l'ensemble des résultats antérieurs à 1991 (RUSSO et THURSTON, 1991 ) indique que chez les salmonidés et les autres espèces de poissons, il y a un impact sur la croissance entre 0,02 et $0,15 \mathrm{mg} / \mathrm{NH}_{3}$ pour une durée d'exposition de 1 à 3 mois. Des seuils de sécurité beaucoup plus bas sont proposés pour l'ensemble des organismes vivants, $0,029 \mathrm{mg} / \mathrm{l}$ (US EPA, 1989), et de 0,021 pour les poissons d'eau douce (EIFAC, 1984). Ils sont estimés en comparant le rapport seuils de toxicité aiguë/chronique pour les espèces les plus sensibles (poissons d'eau douce).

\section{Tableau II}

Comparaison des CL50 chroniques pour une durée d'exposition de 28 jours et de différents seuils de perturbation de la croissance (arrêt, seuil de risque et seuil de sécurité) chez des juvéniles de 4 espèces de poissons d'eau de mer et chez le saumon atlantique en eau douce (35 g) et en eau de mer $(600 \mathrm{~g})$ en fonction de la durée d'exposition.

Table II

Comparative chronic 28-day LC50 and thresholds for growth (No GC, LOEC and NOEC) in juveniles of 4 marine fish species plus Atlantic salmon in fresh water $(35 \mathrm{~g})$ and sea water $(600 \mathrm{~g})$ related to exposure duration.

\begin{tabular}{|c|c|c|c|c|c|c|c|}
\hline Espèce & $\begin{array}{l}\text { Durée } \\
\text { (jour) }\end{array}$ & $\begin{array}{l}\text { Poids } \\
\text { (g) }\end{array}$ & $\begin{array}{l}\text { Seuil de } \\
\text { sécurité } \\
\text { (NOEC) }\end{array}$ & $\begin{array}{l}\text { Seuil de } \\
\text { risque } \\
\text { (LOEC) }\end{array}$ & $\begin{array}{c}\text { Arrêt } \\
\text { croissance } \\
\text { (No GC) }\end{array}$ & $\begin{array}{l}\text { 28-J } \\
\text { CL50 }\end{array}$ & Références \\
\hline \multirow{7}{*}{$\begin{array}{l}\text { Turbot } \\
\text { (Psetta } \\
\text { maxima) }\end{array}$} & 11 & 2.3 & 0,14 & - & 0,90 & - & ALDERSON, 1979 \\
\hline & 20 & 20 & 0,11 & - & 0,70 & - & RASMUSSEN et KORSGAARD, 1996 \\
\hline & 34 & 104 & - & 0,09 & 0,80 & 1.1 & PERSON-LE RUYET et al., 1997a \\
\hline & 42 & 14 & 0,18 & 0,40 & 0,96 & 0,8 & \\
\hline & 28 & 23 & - & 0,21 & 0,81 & 1,0 & \\
\hline & 84 & 50 & 0.14 & 0,33 & 0.73 & 1,2 & PERSON-LE RUYET et al. 1997b \\
\hline & 84 & 72 & - & 0,14 & 0,73 & 1.0 & \\
\hline & & 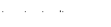 & & & & & \\
\hline $\begin{array}{l}\text { Sole commune } \\
\text { (Solea solea) }\end{array}$ & 42 & $0,6-0,20$ & 0,05 & - & 0,77 & & ALDERSON, 1979 \\
\hline & & & & & & & \\
\hline $\begin{array}{l}\text { Daurade dorée } \\
\text { (Sparus aurata) }\end{array}$ & 20 & 0,3 & 0.27 & 0.47 & & $0.80^{\circ}$ & WAJSBROT et al. 1993 \\
\hline $\begin{array}{c}\text { Bar européen } \\
\text { (Dicentrarchus } \\
\text { labrax) }\end{array}$ & 54 & 2.9 & - & 0,25 & - & 0,90 & LEMARIÉ et al., 1996 \\
\hline $\begin{array}{c}\text { Saumon } \\
\text { atlantique } \\
\text { (Salmo salar) }\end{array}$ & $\begin{array}{l}28 \\
31\end{array}$ & $\begin{array}{c}35 \\
600\end{array}$ & $\begin{array}{l}0,06 \\
0.08\end{array}$ & & & & $\begin{array}{l}\text { FIVELSTAD et al., } 1993 \\
\text { FIVELSTAD et al., } 1995\end{array}$ \\
\hline
\end{tabular}

Les seuils de sécurité en termes de croissance (Tableau II) sont en première approximation (les tailles et les conditions expérimentales n'étant pas strictement comparables) du même ordre chez les juvéniles de bar, daurade et turbot (WAJSBROT et al., 1993 ; RASMUSSEN et KORSGAARD, 1996 ; LEMARIÉ et al., 1996). Chez les salmonidés, les données les plus récentes (FIVELSTAD et al., 1993, 1995) confirment la plus grande sensibilité à long terme du saumon atlantique en comparaison avec les 
poissons marins éprouvés, à l'exception, peut-être, de la sole (ALDERSON, 1979). Elle est toutefois sans commune mesure avec les différences observées en toxicité aiguë (CL50 variant de 1 à 10). Selon l'US EPA, le rapport seuils de toxicité aiguë/chronique serait plus élevé en eau de mer qu'en eau douce, mais il faut convenir qu'il y a un manque évident de données expérimentales sur les seuils de sécurité chez les poissons marins.

\section{FACTEURS AFFECTANT LA TOXICITÉ DE L'AAT}

\subsection{Facteurs environnementaux}

Il existe une littérature abondante sur l'impact des facteurs environnementaux sur la toxicité de l'AAT chez les poissons d'eau douce, synthétisée dans RUSSO et THURSTON (1991). Par contre, en eau de mer, les données disponibles sont très fragmentaires. Un des plus importants est le $\mathrm{pH}$ ambiant. II intervient en modifiant l'équilibre $\mathrm{NH}_{4}{ }^{+}-\mathrm{NH}_{3}$, la proportion de $\mathrm{NH}_{3}$ étant augmentée aux pH élevés. De plus, la toxicité du $\mathrm{NH}_{3}$ et celle du $\mathrm{NH}_{4}{ }^{+}$augmentent lorsque le $\mathrm{pH}$ décroît. Ceci a été mis en évidence pour une gamme de $\mathrm{pH}$ très étendue, 6-10, chez les crustacés et les poissons, en eau douce et en eau de mer et pour des expositions de courte et de longue durée. Pour les poissons marins, les données disponibles chez la sole (ALDERSON, 1979) sont à utiliser avec précaution car les effets d'une acidification du milieu $(\mathrm{pH}, 7,9-6,9)$ semblent varier selon le critère considéré (arrêt de croissance, seuil de risque ou de sécurité en termes de croissance). Il est de même assez difficile de dissocier l'action directe et indirecte de la température et de la salinité. Tout laisse penser que pour la température, il n'y a pas d'effet tant qu'on se situe dans la plage thermique optimale pour la croissance ; en dessous, la sensibilité serait diminuée et au-dessus, elle serait augmentée (ERICKSON, 1985). Pour la salinité, les différences apparentes de réponse entre espèces seraient en rapport avec leur capacité d'osmorégulation. En eau douce, la composition ionique de l'eau $\left(\mathrm{Ca}^{++}, \mathrm{Na}^{+}\right)$modifie de même la sensibilité au $\mathrm{NH}_{3}$. Par ailleurs, il ne fait pas de doute qu'une diminution, même modérée, de la disponibilité en oxygène dissous augmente la sensibilité de tous les organismes au $\mathrm{NH}_{3}$. Chez la daurade dorée, la 96-h CL50 est diminuée de moitié lorsque le $\%$ de saturation en oxygène passe de 85 à $40 \%$; en deçà, l'augmentation de sensibilité est considérablement amplifiée (WAJSBROT et al., 1991). Les effets potentiels d'une sursaturation en oxygène ne sont par contre pas connus; il est de même pour le $\mathrm{CO}_{2}$.

\subsection{Facteurs biologiques}

Nous avons précédemment insisté sur le fait que parmi les poissons élevés, les salmonidés en eau douce et dans une moindre mesure en eau de mer sont les plus sensibles ; les poissons-chats et les poissons marins éprouvés (turbot, daurade, bar) sont les plus résistants. La sensibilité varie aussi selon le stade de développement et la taille des poissons (RUSSO et THURSTON, 1991). Chez les salmonidés, elle est élevée pendant l'embryogenèse, décroît à l'éclosion avec une remontée au passage à l'alimentation exogène qui se poursuit chez les alevins jusqu'à un stade plus ou moins avancé selon les espèces, pour rediminuer pendant la période de grossissement. Chez de nombreuses espèces de poissons marins, les très jeunes larves sont très sensibles au stade du passage à l'alimentation exogène (BROWNELL, 1980).

\subsection{Facteurs liés aux conditions d'exposition}

Les effets bénéfiques d'une pré-acclimatation sont bien connus, nous les avons évoqués précédemment chez le turbot. Des salmonidés soumis occasionnellement ou régulièrement à des pics de $\mathrm{NH}_{3}$, ou exposés à une augmentation progressive de la concentration en $\mathrm{NH}_{3}$ ambiant, sont susceptibles d'acquérir une tolérance plus élevée que des lots non pré-conditionnés. Cependant BROWN et al. (1969) ont montré que, chez la truite (Oncorhynchus mykiss), les effets de l'acclimatation ne sont bénéfiques que pour une 
amplitude et une fréquence de pics de $\mathrm{NH}_{3}$ bien définies. Une stimulation de la croissance dans certaines conditions d'exposition à l'AAT est de même mentionnée, mais non démontrée à long terme. Par ailleurs, il ne fait pas de doute que, pré-acclimatation ou pas, les seuils de tolérance au $\mathrm{NH}_{3}$ diminuent avec la durée d'exposition. L'utilisation de durée d'exposition trop courte entraîne une sous-estimation des seuils de tolérance. II faut aussi avoir à l'esprit les interactions de l'ensemble des facteurs du milieu évoqués dans ce chapitre ainsi que les effets de fluctuations des niveaux d'AAT ambiant sur la sensibilité des poissons.

\section{CONCLUSION}

L'AAT est un neurotoxique du système nerveux central. Dès qu'un poisson se trouve en présence de concentrations anormalement élevées d'AAT, quelle qu'en soit la cause, il y a entrée d'AAT essentiellement sous forme de $\mathrm{NH}_{3}$ et diffusion dans la masse corporelle en moins d'une heure. A partir d'un certain niveau de concentration d'AAT dans le sang et les tissus (variable selon l'espèce et la durée d'exposition), le poisson n'arrive plus à réguler ses fonctions vitales, le seuil de toxicité est atteint. En deçà, il s'adapte et il semble que l'ammoniémie puisse avoir une valeur prédictive des seuils de perturbation de la croissance et des limites de survie chez d'autres espèces comme chez le turbot.

Dans les élevages à haute densité utilisant des circuits fermés, l'AAT ambiant et le $\mathrm{NH}_{3}$, la forme la plus toxique, peuvent atteindre des concentrations défavorables à la bonne réussite des élevages. Pour des durées d'exposition d'un mois, les seuils de toxicité chronique sont relativement élevés $\left(0,8-1 \mathrm{mg} / \mathrm{NH}_{3}\right)$ et inhabituels. Par contre il faut être vigilant pour se situer en deçà des seuils de risque qui se situent autour de $0,11 \mathrm{mg} / 1 \mathrm{NH}_{3}$ pour des périodes d'élevage n'excédant pas 3 mois. II est difficile de prédire des seuils de risque avec une sécurité suffisante à partir de tests de croissance de moins d'un mois et en l'absence de répétition. Il est aussi risqué de procéder par analogie avec d'autres espèces, compte tenu de différences très grandes de sensibilité entre espèces. Les poissons marins dont le turbot sont globalement du type poisson-chat, c'est-à-dire relativement tolérants à l'AAT par rapport aux salmonidés.

La toxicité de l'AAT est dépendante de facteurs environnementaux $(\mathrm{pH}$, température et salinité) et biologiques (espèce, taille et stade de développement) et elle peut être modulée par les conditions d'exposition (durée d'exposition et pré-acclimatation). Le degré de toxicité des deux formes d'AAT $\left(\mathrm{NH}_{3}\right.$ et $\left.\mathrm{NH}_{4}{ }^{+}\right)$varie avec le $\mathrm{pH}$ ambiant et par ailleurs, les $\mathrm{pH}$ acides augmentent la sensibilité des poissons aux deux formes d'AAT $\left(\mathrm{NH}_{3}\right.$ et $\left.\mathrm{NH}_{4}{ }^{+}\right)$. Ceci nous amène à considérer que dans un contexte d'intensification des élevages de poissons marins, l'étude de l'interaction $\mathrm{pH}-\mathrm{NH}_{3}$ (ou du couple $\mathrm{pH}-\mathrm{CO}_{2}$ et $\mathrm{NH}_{3}$ ) est une priorité. Par contre, en milieu confiné (transport de poissons vivants) l'interaction $\mathrm{O}_{2}-\mathrm{NH}_{3}$ devient décisive.

En définitive, on peut considérer que même les plus faibles concentrations en $\mathrm{NH}_{3}$ ambiant ne sont pas, au terme d'un cycle d'élevage long (le plus souvent 2 années), neutres pour le poisson, malgré de bonnes capacités d'adaptation à court terme.

\section{REMERCIEMENTS}

Les auteurs tiennent particulièrement à remercier l'ensemble des personnes de la Direction des Ressources Vivantes du Centre IFREMER de Brest ayant participé aux travaux de recherche sur la toxicité de l'AAT chez le turbot ou assuré la mise en forme de la synthèse des résultats : H. CHARTOIS, E. DESBRUYERES, A. LE ROUX, B. PETTON, L. QUEMENER, N. ROSSIGNOL et A. SEVERE. Ce travail a été réalisé dans le cadre du Programme de Recherche "Régulation de la croissance chez les poissons d'intérêt aquacole ". 


\section{BIBLIOGRAPHIE}

ABEL P.D., AXIAK V., 1991. Ecotoxicology and the marine environment. Ellis Horwood, London, $264 \mathrm{p}$.

ALABASTER J.S., SHURBEN D.G., MALLETT M.J., 1983. The acute lethal toxicity of mixtures of cyanide and ammonia to smolts of salmon, Salmo salar L., at low concentrations of dissolved oxygen. J. Fish Biol., 22, 215-222.

ALDERSON R., 1979. The effect of ammonia on the growth of juvenile Dover sole, Solea solea (L.) and turbot, Scophthalmus maximus (L.). Aquaculture, 17, 291-309.

AMINOT A., 1983. Dosage de l'azote ammoniacal. In Manuel des analyses chimiques en milieu marin, CNEXO, BNDO/Documentation éditeurs, 107-118.

ARILLO A., MARGIOCCO C., MELODIA F., MENSI P., SCHEMONE G., 1981. Ammonia toxicity mechanism in fish : studies on rainbow trout (Salmo gairdneri Rich.). Ecotoxicol. Environ. Safety, 5, 316-328.

ARMSTRONG D.A., CHIPPENDALE D., KNIGHT A.W., COLT J.E., 1978. Interaction of ionized and un-ionized ammonia on short-term survival and growth of prawn larvae, Macrobrachium rosenbergii. Biol. Bull., 154, 15-31.

BANERJEE S., BHATTACHARYA S., 1995. Histopathological changes induced by chronic nonlethal levels of elsan, mercury, and ammonia in the small intestine of Channa punctatus (Bloch). Ecotoxicol. Environ. Safety, 31, 62-68.

BIANCHINI A., WASIELESKY Jr., MIRANDAFIHO K.C., 1996. Toxicity of nitrogenous compounds to juveniles of flatfish Paralichthys orbignyanus. Bull. Environ. Contam. Toxicol., 56, 453-459.

BROWN V.M., JORDAN D.H.M., TITLER B.A., 1969. The acute toxicity to rainbow trout of fluctuating concentrations and mixtures of ammonia, phenol and zinc. J. Fish Biol., $1,1-9$.

BROWNELL C.L., 1980. Water quality requirements for first-feeding in marine fish larvae. 1 - Ammonia, nitrite and nitrate. J. Exp. Mar. Biol. Ecol., 44, 269-283.

BURY N.R., EDDY F.B., CODD G.A., 1995. The effects of the cyanobacterium Microcystis aeruginosa, the cyanobacterial hepatotoxin microcystin-LR, and ammonia on growth rate and ionic regulation of brown trout. J. Fish Biol., 46, 1042-1054.

CAMERON J.N., HEISLER N., 1983. Studies of ammonia in the rainbow trout : physicochemical parameters, acid-base behavior and respiratory clearance. J. Exp. Biol., 105, 107-125.

CAMERON J.N., 1986. Responses to reversed $\mathrm{NH}_{3}$ and $\mathrm{NH}_{4}{ }^{+}$gradients in a teleost (Ictalurus punctatus), an elasmobranch (Raja erinacea) and a crustacean (Callinectes sapidus) : evidence for $\mathrm{NH}_{4}{ }^{+} / \mathrm{H}^{+}$exchange in the teleost and the elasmobranch. J. Exp. Zool., 239, 183-195.

CHO Y.J., KIM Y.Y., LEE N.G., CHOI Y.J., 1994. Basic studies on developing equipment for waterless transportation of live fish. Bull. Korean Fish. Soc., 27, 501-508.

COOPER A.J.L., PLUM F., 1987. Biochemistry and physiology of brain ammonia. Physiological reviews, 67, 440-519.

COVES D., GASSET E., 1996. Intensive recirculating production system in seabass, Dicentrarchus labrax. Future trends of aquaculture development in Eastern Europe. Handbook of short communications and national reports, Budapest, Hungary, 22-23.

CRIPPS S.J., POXTON M.G., 1992. A review of the design and performance of tanks relevant to flattish culture. Aquacult. Eng., 11, 71-91.

DOSDAT A., GAUMET F., CHARTOIS H., 1994. Marine aquaculture effluent monitoring : methodological approach to the evaluation of nitrogen and phosphorus excretion by fish. Aquacult. Eng., 14, 59-84. 
DOSDAT A., SERVAIS F., METAILLER R., HUELVAN C., DESBRUYERES E., 1996. Comparison of nitrogenous losses in five teleost fish species. Aquaculture, 141, 107-127.

EIFAC, 1984. Water quality criteria for European freshwater fish. Report on nitrite and ammonia. EIFAC Technical Paper, 46, FAO, Rome, Italy, $12 \mathrm{p}$.

ERICKSON R.J., 1985. An evaluation of mathematical models for the effects of $\mathrm{pH}$ and temperature on ammonia toxicity to aquatic organisms. Water Res., 19, 1047-1058.

EVANS D.H., MORE K.J., ROBBINS S.L., 1989. Modes of ammonia transport across the gill epithelium of the marine teleost fish Opsanus beta. J. Exp. Biol., 144, 339-356.

FIVELSTAD S., KALLEVIK H., IVERSEN H.M., MORETRO T., VAGE K., BINDE M., 1993. Sublethal effects of ammonia in soft water on Atlantic salmon smolts at a low temperature. Aquaculture International, 1, 157-169.

FIVELSTAD S., SCHWARZ J., STROMSNES H., 1995. Sublethal effects and safe levels of ammonia in seawater for Atlantic Salmon postsmolts (Salmo salar L.). Aqua. Eng., $14,271-280$.

GUILLEN J.L., ENDO M., TURNBULL J.F., KAWATSU H., RICHARDS R.H., AOKI T., 1994. Skin responses and mortalities in the larvae of Japanese croaker exposed to ammonia. Fisheries Sciences, 60, 547-550.

HANDY R.D., POXTON M.G., 1993. Nitrogen pollution in mariculture : toxicity and excretion of nitrogenous compounds by marine fish. Reviews in Fish Biol. Fish., 3 , 205-241.

HAYWOOD G.P., 1983. Ammonia toxicity in teleost fishes : a review. Can. Tech. Rep. Fish. Aquat. Sci., 1177, 1-35.

HOEKSTRA J.A., VAN EWIJK P.H., 1993. The bounded effect concentration as an alternative to the NOEC. In The Science of the Total Environment, Supplement, Elsevier Science Publishers B.V., Amsterdam, 705-711.

JOHANSSON O., WEDBORG W., 1980. The ammonia-ammonium equilibrium in seawater at temperature between 5 and $25^{\circ} \mathrm{C}$. J. Solut. Chem., 91, 37-44.

JONES A., BROWN J.A.G., DOUGLAS M.T., THOMPSON S.J., WHITFIELD R.J., 1981. Progress towards developing methods for the intensive farming of turbot (Scophthalmus maximus L.) in cooling water from a nuclear power station. In Proc. World Symp. Aquaculture in Heated Effluents and Recirculation Systems, Rosenthal $\mathrm{H}$. and Tiews K. eds, 2, Berlin, 481-496.

KNOPH M.B., 1992. Acute toxicity of ammonia to Atlantic salmon (Salmo salar L.) parr. Comp. Biochem. Physiol., 101, 272-282.

KNOPH M., THORUD K., 1996. Toxicity of ammonia to Atlantic salmon (Salmo salar L.) in seawater. Effects on plasma osmolarity, ion, ammonia, urea and glucose levels and haematologic parameters. Comp. Biochem. Physiol., 113, 375-381.

KOOIJMAN S.A.L.M., BEDAUX J.J.M., 1996. Analysis of toxicity tests on fish growth. Water Res., 30, 1633-1644.

LANG T., PETERS G., HOFFMANN R., MEYER E., 1987. Experimental investigations on the toxicity of ammonia : effects on ventilation frequency, growth, epidermal mucous cells, and gill structure of rainbow trout Salmo gairdneri. Dis. aquat. Org., 3, 159-165.

LEMARIÉ G., COVES D., DUTTO G., GASSET E., PERSON-LE RUYET J., 1996. Chronic toxicity of ammonia for European seabass (Dicentrarchus labrax) juveniles. In Applied environmental physiology of fishes symposium proceedings, Swanson C., Young P. and Mackinlay D. editors, International congress on the biology of fishes, San Francisco State University, July 14-18, 1996, American fisheries society, Bethesda, 65-76. 
LUMSDEN J.S., WRIGHT P., DERKSEN J., BYRNE P.J., FERGUSON H.W., 1993. Paralysis in farmed Artic char (Salvelinus alpinus) associated with ammonia toxicity. Vet. Record, 133, 422-423.

LYGREN E., 1994. Culture in recirculation systems. EAS (European Aquaculture Society) Spec. Publ., 22, 93-104.

MEADE J.W., 1985. Allowable ammonia for fish culture. Prog. Fish Cultur., 47, 135-145.

MOMMSEN T.P., WALSH P.J., 1992. Biochemical and environmental perspectives on nitrogen metabolism in fishes. Experientia, 48, 583-593.

OGATA H., MURAI T., 1988. Changes in ammonia and amino acids levels in the erythrocytes and plasma of carp, Cyprinus carpio, during passage through the gills. J. Fish Biol., 33, 471-479.

OIESTAD V., 1995. Shallow raceways as the basis for industrial production centres of seafood. EAS (European Aquaculture Society) Spec. Pub., 23, 304-305.

OLSON K.R., FROMM P.O., 1971. Excretion of urea by two teleosts exposed to different concentrations of ambient ammonia. Comp. Biochem. Physiol., 40, 999-1007.

PALEY R.K., TWICHTEN I.D., EDDY F.B., 1993. Ammonia, $\mathrm{Na}^{+}, \mathrm{K}^{+}$and $\mathrm{Cl}$ levels in rainbow trout yolk sac fry in response to external ammonia. J. Exp. Biol., 18, 273-284.

PERSON-LE RUYET J., 1993. L'élevage du turbot en Europe. Pis. Franc., 112, 5-22.

PERSON-LE RUYET J., CHARTOIS H., DESBRUYERES E., THOMAS J.L., 1994. Ammonia acute toxicity in turbot (Scophthalmus maximus) juveniles. EAS (European Aquaculture Society), Spec. Publ., 22, 283-291.

PERSON-LE RUYET J., CHARTOIS H., QUEMENER L., 1995. Comparative acute ammonia toxicity in marine fish and plasma ammonia response. Aquaculture, 136, 181-194.

PERSON-LE RUYET J., DELBARD C., CHARTOIS H., LE DELLIOU H., 1997a. Toxicity of ammonia to turbot juveniles : 1 - Effects on survival, growth and food utilisation. Aquat. Living Resour., 10, 307-314.

PERSON-LE RUYET J., GALLAND R., LE ROUX A., CHARTOIS H., 1997b. Chronic ammonia toxicity in juvenile turbot (Scophthalmus maximus). Aquaculture, 154, 155-171.

PERSON-LE RUYET J., BOEUF G., ZAMBONINO INFANTE J., HELGASON S., LE ROUX A., 1998. Short-term physiological changes in turbot and seabream juveniles exposed to exogenous ammonia. Comp. Biochem. Physiol., 119, 511-518.

RANDALL D.J., WRIGHT P.A., 1987. Ammonia distribution and excretion in fish. Fish Physiol. Biochem., 3, 107-120.

RASMUSSEN R.S., KORSGAARD B., 1996. The effect of external ammonia on growth and food utilization of juvenile turbot (Scophthalmus maximus L.). J. Exp. Mar. Biol. Ecol., 205, 35-48.

RUFFIER P.J., BOYLE W.C., KLEINSCHMIDT J.K., 1981. Short-term acute bioassays to evaluate ammonia toxicity and effluent standards. Journal WPCF. Water Pollution Contz. Fed., 53, 367-377.

RUSSO R.C., THURSTON R.V., 1991. Toxicity of ammonia, nitrite and nitrate to fishes. Aquaculture and water quality. In Advances in world aquaculture, Brune $E$. and Tomasso J.R. eds, WAS Publ., 3, 58-89.

SAHA N., RATHA B.K., 1990. Alterations in excretion pattern of ammonia and urea in a freshwater air-breathing teleost, Heteropneustes fossilis (Bloch) during hyper-ammonia stress. Indian J. Exp. Biol., 28, 597-599.

SAHA T.K., ARYA M.B., DAST A.B., 1994. Atlerations in the patterns of nitrogenous excretion in a freshwater air-breathing teleost, Channa punctatus (Bloch), during ammonia-stress. Ind. J. Exp. Biol., 32, 196-199.

SAHA T.K., DAS N.P., ARYA M.B., DAST A.B., 1995. Effect of ammonia-stress on the tissue levels of nitrogenous biomolecules of the air-breathing fish, Channa punctatus (Bloch). Ind. J. Exp. Biol., 33, 943-952. 
SALIN D., WILLIOT P., 1991. Acute toxicity of ammonia to Siberian sturgeon Acipenser baeri. In Acipenser, Williot P. editor, Cemagref Publ., 153-167.

SCHENONE G., ARILLO A., MARGIOCCO C., MELODIA F., MENSI P., 1982. Biochemical bases for environmental adaptation of goldfish (Carassius auratus L.) : resistance to ammonia. Ecotox. Environ. Safety, 6, 479-488.

THURSTON R.V., RUSSO R.C., VINOGRADOV G.A., 1981. Ammonia toxicity to fishes. The effects of $\mathrm{pH}$ on the toxicity of the un-ionized ammonia species. Environ. Sci. Tech., $15,837-840$.

TOMASSO J.R., 1994. Toxicity of nitrogenous wastes to aquaculture animals. Rev. Fish. Sci., 2, 291-314.

TORANZO A.E., BARJA J.L., DEVESA S., 1994. An overview of the main infectious problems in cultured turbot : present status and future necessities. EAS (European Aquaculture Society), Spec. Publ., 22, 106-126.

UNEP, 1989. Test of the acute lethal toxicity of pollutants to marine fish and invertebrates. References methods for marine pollutions studies, No 43, $27 \mathrm{p}$.

US EPA (United States Environmental Protection Agency), 1989. Ambient water quality criteria for ammonia (salt-water), EPA 440/5-88-04, Office of Water Regulations and Standards Criteria and Standards Division, Washington, DC, $28 \mathrm{p}$.

WAJSBROT N., GASITH A., KROM M.D., POPPER D.M., 1991. Acute toxicity of ammonia to juvenile gilthead seabream Sparus aurata under reduced oxygen levels. Aquaculture, 92, 277-288.

WAJSBROT N., GASITH A., DIAMANT A., POPPER D.M., 1993. Chronic toxicity of ammonia to juvenile gilthead seabream Sparus aurata and related histophathological effects. J. Fish Biol., 43, 321-328.

WEIRICH C.R., TOMASSO J.R., SMITH T.I.J., 1993. Toxicity of ammonia and nitrite to sunshine bass in selected environments. J. Aquat. Anim. Health, 5, 64-72.

WILSON R.W., TAYLOR E.W., 1992. Transbranchial ammonia gradients and acid-base responses to high external ammonia concentration in rainbow trout (Oncorhynchus mykiss) acclimated to different salinities. J. Exp. Biol., 166, 95-112.

WILSON R.W., WRIGHT P.M., MUNGER S., WOOD C.M., 1994. Ammonia excretion in freshwater rainbow trout (Oncorhynchus mykiss) and the importance of gill boundary layer acidification : lack of evidence for $\mathrm{Na}^{+} / \mathrm{NH}_{4}{ }^{+}$exchange. J. Exp. Biol., 191, 37-58.

WISE D.J., WEIRICH C.R., TOMASSO J.R., 1989. Toxicity of ammonia to red drum Sciaenops ocellatus fingerlings with information on uptake and depuration. J. World Aquacult. Soc., 20, 188-192.

WOOD C.M., 1993. Ammonia and urea metabolism and excretion. In The Physiology of Fishes, Evans D.H. editor, 379-425.

WRIGHT P.A., WOOD C.M., 1985. An analysis of branchial ammonia excretion in the freshwater rainbow trout : effects of environmental $\mathrm{pH}$ change and sodium uptake blockage. J. Exp. Biol., 114, 329-353.

WRIGHT P.A., RANDALL D.J., PERRY S.F., 1989. Fish gill water boundary layer : a site of linkage between carbon dioxide and ammonia excretion. J. Comp. Physiol., 158, 627-635.

WRIGHT P.A., 1993. Nitrogen excretion and enzyme pathways for ureagenesis in freshwater tilapia (Oreochromis niloticus). Physiol. Zool., 66, 881-901.

WRIGHT P.A., 1995. Nitrogen excretion : three end products, many physiological roles. J. Exp. Biol., 198, 273-281. 